TOPOLOGICAL ALGEBRAS, THEIR APPLICATIONS,

AND RELATED TOPICS

BANACH CENTER PUBLICATIONS, VOLUME 67

INSTITUTE OF MATHEMATICS

POLISH ACADEMY OF SCIENCES

WARSZAWA 2005

\title{
PONTRYAGIN DUALITY AND TOPOLOGICAL ALGEBRAS
}

\author{
SERGEI S. AKBAROV \\ Department of Mathematics, Russian Institute of Scientific and Technical Information \\ Usievicha 20, Moscow, 125190 Russia \\ E-mail:akbarov@mail.com
}

As is known, the Pontryagin duality is a traditional object of investigations in Group Theory. Another application of this idea was found quite recently in the theory of topological algebras and is known as stereotype theory. A detailed description of this theme was presented in the author's survey [1] and in his big paper [2] containing all the exact formulations and the accurate proofs.

This note has the publicity aims: we would like to discuss here some results of [2] concentrating our attention mostly on the motives and giving a more popular form to the exposition. We announce, in addition, some new results and list some open problems.

Let us start with the following philosophical question:

\section{What is the difference between the Banach and the topological algebras?}

To explain our idea, we would like to suggest the following

ExERCISE A. Give a definition of topological algebra and topological module such that the following conditions hold:

1) all the topological modules are topological vector spaces and satisfy some standard condition of completeness (this is important for constructing the integral theory);

2) the multiplication operations are continuous in some reasonable sense;

3 ) if $X$ is a topological module over a topological algebra $A$, then the $\operatorname{ring} \operatorname{End}_{A}(X)$ of all endomorphisms of $X$ over $A$ can also be endowed with the structure of topological algebra with respect to your definition.

If you think about this, you will see that neither algebras with jointly continuous multiplication, nor the ones with separately continuous one can be suggested as answers.

2000 Mathematics Subject Classification: Primary 46H05.

Key words and phrases: approximation property, bicommutant theorem, enriched category, group algebra, Hopf algebra, stereotype algebra, stereotype space.

The paper is in final form and no version of it will be published elsewhere. 
This can be unexpected, but the only elementary answer coming to mind here is: 'Banach algebras and Banach modules'. This follows from the well-known categorial properties of Banach spaces:

THEOREM 1. The class $\mathfrak{B a n}$ of Banach spaces has the following categorial properties:

(i) $\mathfrak{B a n}$ is a closed symmetrical monoidal category;

(ii) Banach algebras are exactly monoids in $\mathfrak{B a n}$;

(iii) for every Banach algebra $A$ the classes $A \mathfrak{B a n}$ and $\mathfrak{B a n}_{A}$ of left and right Banach modules over $A$ are enriched categories over $\mathfrak{B a n}$.

Here we give the informal explanation of these words (the accurate definitions can be found in the handbook [5] or in the original monograph [12]):

$\mathbf{1}^{*}$. The phrase about symmetrical monoidal category means that $\mathfrak{B a n}$ has a natural (projective) tensor product $\widehat{\otimes}$ satisfying the following natural identities:

$$
X \widehat{\otimes}(Y \widehat{\otimes} Z) \cong(X \widehat{\otimes} Y) \widehat{\otimes} Z, \quad X \widehat{\otimes} Y \cong Y \widehat{\otimes} X, \quad \mathbb{C} \widehat{\otimes} X \cong X \cong X \widehat{\otimes} \mathbb{C} .
$$

$\mathbf{2}^{*}$. The words 'closed category' mean that for each Banach spaces $X, Y \in \mathfrak{B a n}$ the space of morphisms $\operatorname{Mor}(X, Y)$ can be endowed naturally with the structure of Banach space $\operatorname{Mor}(X, Y) \in \mathfrak{B a n}$, and the following natural identity holds:

$$
\operatorname{Mor}(X \widehat{\otimes} Y, Z)=\operatorname{Mor}(X, \operatorname{Mor}(Y, Z)) \text {. }
$$

$\mathbf{3}^{*}$. An algebra in $\mathfrak{B a n}$ is a monoid $(A, \alpha, \varepsilon)$ in $\mathfrak{B a n}$, i.e. an object $A$ with chosen morphisms $\alpha: A \widehat{\otimes} A \rightarrow A$ (multiplication) and $\varepsilon: \mathbb{C} \rightarrow A$ (unit) such that the following diagrams are commutative:
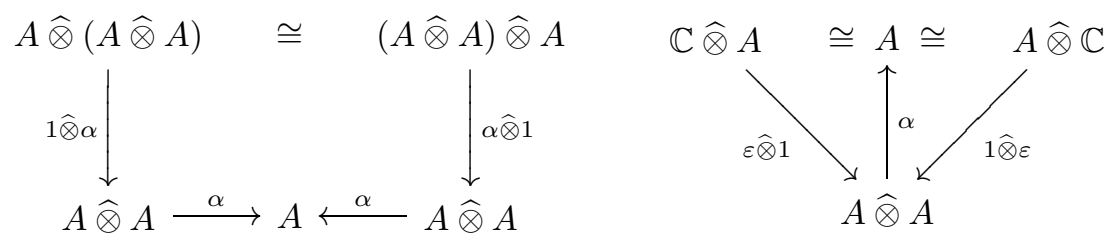

A morphism of algebras $\varphi:(A, \alpha, \varepsilon) \rightarrow(B, \beta, v)$ is a morphism of monoids in $\mathfrak{B a n}$, i.e. $\varphi: A \rightarrow B$ such that
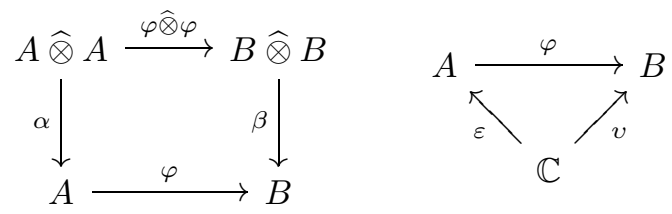

$4^{*}$. A left $A$-module in $\mathfrak{B a n}$ is an object $X$ with a morphism $\mu: A \widehat{\otimes} X \rightarrow X$ (multiplication by elements of $A$ ) such that
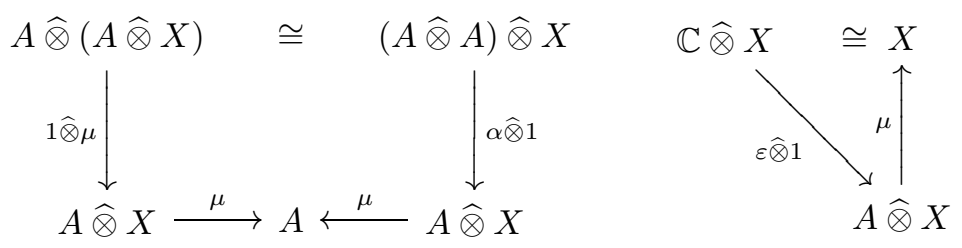
A morphism of $A$-modules $\varphi:\left(X, \mu_{X}\right) \rightarrow\left(Y, \mu_{Y}\right)$ is a morphism $\varphi: X \rightarrow Y$ in $\mathfrak{B a n}$ such that

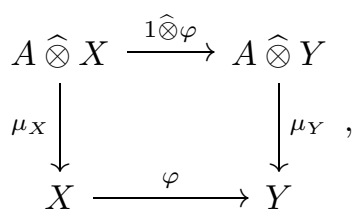

${ }_{A} \mathfrak{B a n}$ is the category of left $A$-modules.

$\mathbf{5}^{*}$. The category of right $A$-modules $\mathfrak{B a n} \mathfrak{n}_{A}$ can be defined in the same way.

6*. The words ' $A \mathfrak{B a n}$ and $\mathfrak{B a n}_{A}$ are enriched categories over $\mathfrak{B a n}$ ' mean that for each $A$-modules $X, Y \in{ }_{A} \mathfrak{B a n}$ (respectively, $X, Y \in \mathfrak{B a n}_{A}$ ) the set of morphisms can be endowed naturally with the structure of Banach space $\operatorname{Mor}_{A}(X, Y) \in \mathfrak{B a n}$, and the operation of composition $(\varphi, \psi) \mapsto \varphi \circ \psi$ can be extended to a morphism in $\mathfrak{B a n}$

$$
\operatorname{Mor}_{A}(Y, Z) \widehat{\otimes} \operatorname{Mor}_{A}(X, Y) \longrightarrow \operatorname{Mor}_{A}(X, Z) \text {. }
$$

2. Category $\mathfrak{S t}_{\mathfrak{t}}$ of stereotype spaces. It turns out that the category $\mathfrak{B a n}$ of Banach spaces is not the only one that provides the correct definition for topological algebras and topological modules in the sense of Exercise A. Another possibility was described in the author's works $[1,2]$, and the topological vector spaces lying in its fundament are called stereotype spaces. Their definition is as follows.

Let $X$ be a locally convex space over $\mathbb{C}$. Denote by $X^{\star}$ the space of all linear continuous functionals

$$
f \in X^{\star} \Rightarrow f: X \rightarrow \mathbb{C}
$$

and endow it with the topology of uniform convergence on totally bounded sets in $X$. Consider the natural mapping

$$
i: X \rightarrow\left(X^{\star}\right)^{\star} \quad \mid \quad i(x)(f)=f(x), \quad x \in X, f \in X^{\star} .
$$

Definition. $X$ is stereotype if the mapping $i: X \rightarrow\left(X^{\star}\right)^{\star}$ is an isomorphism of locally convex spaces (i.e., linear homeomorphism):

$$
X \cong\left(X^{\star}\right)^{\star} \text {. }
$$

It is not difficult to recognize here one of the modifications of Pontryagin duality for topological Abelian groups (see details in [3]). The following criterion makes this notion more intelligible.

Criterion. $X$ is stereotype if and only if the following two conditions are fulfilled:

(i) $X$ is pseudocomplete: every totally bounded Cauchy net converges in $X$;

(ii) $X$ is pseudosaturated: every closed, convex, balanced capacious set $D$ in $X$ is a neighborhood of zero. ( $D$ is said to be capacious in $X \Longleftrightarrow$ for every totally bounded set $S \subset X$ there exists a finite set $A \subset X$ such that $S \subset D+A$.)

The first important property of stereotype spaces is that they form an unexpectedly wide class - they contain all quasicomplete barrelled spaces, and as a consequence, all Fréchet spaces, and all reflexive spaces (in the sense of usual reflexivity condition for 
locally convex spaces). So it will not be a serious exaggeration if we say that all the spaces actually used in analysis are stereotype:

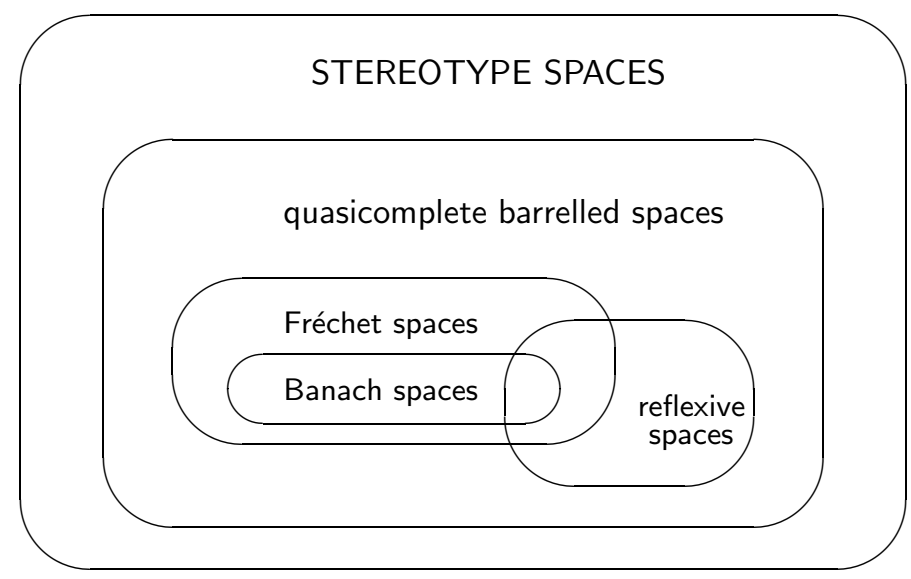

The class $\mathfrak{S t e}_{\mathfrak{e}}$ of stereotype spaces forms a category with linear continuous maps as morphisms. It has the following properties:

$1^{0}$. Ste is pre-Abelian (i.e. additive category, where every morphism has kernel, cokernel, image and coimage);

$2^{0}$. Ste is complete and co-complete (i.e. every projective and every injective system have a limit);

$3^{0}$. Ste is autodual with respect to the functor $X \mapsto X^{\star}$ of passing to the dual space.

Another important fact is that in the category $\mathfrak{S t e}$ there is a natural way to endow the set $\operatorname{Mor}(X, Y)$ of morphisms (i.e., linear continuous maps) $\varphi: X \rightarrow Y$ with the structure of stereotype space:

$$
\forall X, Y \in \mathfrak{S t e} \operatorname{Mor}(X, Y) \in \mathfrak{S t e} .
$$

This permits one to define two natural tensor products in Ste:

Definition. For any two stereotype spaces $X, Y \in \mathfrak{S t e}$ their stereotype projective tensor product $X \circledast Y$ and stereotype injective tensor product $X \odot Y$ are defined by the following equalities:

$$
X \circledast Y:=\operatorname{Mor}\left(X, Y^{\star}\right)^{\star}, \quad X \odot Y:=\operatorname{Mor}\left(X^{\star}, Y\right) .
$$

The following examples justify these definitions:

1) if $X, Y$ are Fréchet spaces, then the stereotype projective tensor product $X \circledast Y$ coincides with the classical projective tensor product $X \hat{\otimes} Y$;

2) if $X, Y$ are Fréchet spaces and $X$ or $Y$ has the classical approximation property, then the stereotype injective tensor product $X \odot Y$ coincides with the classical injective tensor product $X \check{\otimes} Y$.

Stereotype tensor products satisfy the following natural identities: 


$$
\begin{aligned}
\mathbb{C} \circledast X \cong X \cong X \circledast \mathbb{C}, & \mathbb{C} \odot X \cong X \cong X \odot \mathbb{C}, \\
X \circledast Y \cong Y \circledast X, & X \odot Y \cong Y \odot X, \\
(X \circledast Y) \circledast Z \cong X \circledast(Y \circledast Z), & (X \odot Y) \odot Z \cong X \odot(Y \odot Z), \\
(X \circledast Y)^{\star} \cong Y^{\star} \odot X^{\star}, & (X \odot Y)^{\star} \cong Y^{\star} \circledast X^{\star}, \\
\operatorname{Mor}(X \circledast Y, Z) \cong \operatorname{Mor}(X, \operatorname{Mor}(Y, Z)), & \operatorname{Mor}(X, Y \odot Z) \text { Mor }(X, Y) \odot Z .
\end{aligned}
$$

TheOREM 2. (Ste, $\circledast)$ is a closed symmetrical monoidal category.

Let $A$ be a $\circledast$-algebra and ${ }_{A} \mathfrak{S t e}, \mathfrak{S t e}_{A}$ denote the categories of all left and right stereotype modules over $A$. Like in the case of $\mathfrak{S} \mathfrak{t e}$, it turns out that in ${ }_{A} \mathfrak{S t e}$ and $\mathfrak{S t e}_{A}$ there is a natural way to endow the set $\operatorname{Mor}_{A}(X, Y)$ of morphisms $\varphi: X \rightarrow Y$ with the structure of stereotype space:

$$
\forall X, Y \in{ }_{A} \mathfrak{S t e}_{\mathfrak{e}} \quad\left(\forall X, Y \in \mathfrak{S t e}_{A}\right) \quad \operatorname{Mor}_{A}(X, Y) \in \mathfrak{S t e} .
$$

TheOREm 3. For every $\circledast$-algebra $A$ the categories ${ }_{A} \mathfrak{S t e}$ and $\mathfrak{S t e}_{A}$ are enriched categories over Ste.

3. View of the whole functional analysis from the stereotype theory. Theorems 2 and 3 mean that the class $\mathfrak{S t e}$ of stereotype spaces gives a natural solution for Exercise $\mathrm{A}$, and, as a consequence, we may expect that there must be a profound "theory of stereotype topological algebras", no less deep than the classical Banach theory. Indeed, there is much evidence for this, but before presenting it, let us share the whole impression of the arising "new theory".

It can be expressed in the following comparison.

Imagine that you write a textbook on Mathematical Analysis and, being impressed by the debates of the previous century, you remove the axiom of choice and/or all the "nonconstructive proofs" from your text. It is well-known that Analysis will not die in this situation: many notions, like limit, derivative, integral and many classical theorems can be preserved. But the changes will be that

- first, many classical notions become split into non-equivalent ones (for example, the limit in the sense of Cauchy may not be the same as the limit in the sense of Heine - see [4]; similarly, the notion of fundamental sequence can have many different formalizations, like 'quasifundamental', 'pseudofundamental'—see [13], etc.),

- and, second, the connections between the classical notions will be impoverished: many informative results will disappear (making the way for the results describing the connections between the different formalizations of classical notions), while the preserved ones will become overgrown with numerous unusual supplementary assumptions putting serious obstacles in the perception of the proposition.

As a result, all your Mathematical Analysis will be turned into a long list of counterexamples with some rare disseminations of classical theorems hardly recognizable, in addition, because of their unusually bulky formulations.

On the contrary, if you add again the axiom of choice and allow your proofs to be "nonconstructive", then the variations in the understanding of classical notions disappear, the formulations of theorems become more clean and simple, and the "latent" connections between the notions become visible again. 
A very similar situation takes place in the stereotype theory. If you consider the stereotype algebras and modules (as we told before this does not put any real restrictions on the theory, because of the width of the class $\mathfrak{S t e}$ ), the necessity to study different formalizations of the notions falls away, many counterexamples disappear, the definitions and the formulations are simplified, and the "latent" connections come to light.

In short, the idea we try to suggest the reader is that the stereotype theory is to the classical theory of topological algebras as Classical Analysis is to Constructive Analysis:

$\frac{\text { Stereotype Theory }}{\text { Classical Theory of Topological algebras }}=\frac{\text { Classical Mathematical Analysis }}{\text { Constructive Mathematical Analysis }}$

We shall try to illustrate this with two examples - the stereotype analogues of the notions of group algebra and approximation property. We can visualize our plan in the following table:

\begin{tabular}{|c|c|c|c|}
\hline & Classical notion & Latent notions & Latent connections \\
\hline 1 & group algebras & reflection & $\begin{array}{ll}\text { - } & \text { reflections } \leftrightarrow \\
& \leftrightarrow \text { irreducible representations }\end{array}$ \\
\hline 2 & $\begin{array}{l}\text { approximation } \\
\text { property } \\
\text { (A.P.) }\end{array}$ & $\begin{array}{c}\text { A.P. } \\
\text { for modules }\end{array}$ & $\begin{array}{l}\text { - } \text { A.P. is inherited by tensor products } \\
\text { and by the spaces of operators } \\
\text { - A.P. } \leftrightarrow \text { "absolute homology theory" } \\
\text { - A.P. } \leftrightarrow \text { reflections } \\
\text { - A.P. }+ \text { reflections } \leftrightarrow \\
\leftrightarrow \text { bicommutant theorems }\end{array}$ \\
\hline
\end{tabular}

4. Notion 1: group algebras. If $G$ is a finite group, then its group algebra $k G$ over a field $k$ can be defined as the one whose actions on a vector space $X$ over $k$ are equivalent to the actions of the group $G$ on $X$. A very important observation here is that $k G$ has a natural structure of Hopf algebra with respect to the algebraic tensor product $\otimes$ - this fact lies at the foundations of the informal idea used in the theory of Quantum Groups [7]: every Hopf algebra $H$ should be considered as a "generalized group algebra" $k G$ of some "generalized group" $G$.

The construction of group algebra has many generalizations in analysis, but the typical obstacles here are that

1) usually it is not easy to indicate the class of vector spaces $X$ and the class of actions in such a way that the action of $G$ on $X$ corresponds naturally to the action of $k G$ on $X$, and

2 ) if, nevertheless, we had already defined the group algebra $k G$, it is not easy to explain in what sense it is a Hopf algebra.

This leads to the situation similar with that one we mentioned above when discussing Constructive Analysis: on the one hand, there are too many ways for defining group algebras for a given class of groups, and on the other hand, the constructions we obtain look too complicated and inadequate.

To clarify this, let us recall the standard 
Constructions of group algebras in Analysis:

1. Suppose $G$ is a locally compact group. What class of topological vector spaces $X$ over $\mathbb{C}$ should we choose, what construction of the group algebra $\mathbb{C} G$ should we indicate, and how should we define continuous actions of $G$ and $\mathbb{C} G$ on $X$ to ensure the one-to-one correspondence between the actions of $G$ and $\mathbb{C} G$ ?

One possible answer was suggested by G. L. Litvinov in [14]. He defined the group algebra $\mathbb{C} G$ as the algebra $\mathcal{M}_{\sigma}(G)$ of measures on $G$ with compact support endowed with the weak topology generated by the space $\mathcal{C}(G)$ of continuous functions on $G$. Then the action of $\mathcal{M}_{\sigma}(G)$ on $X$ by $X^{\prime}$-weakly continuous operators becomes equivalent to the action of $G$ on $X$ satisfying the following exotic conditions of continuity:

1) all the matrix elements of this action are continuous, i.e. for each $x \in X$ and $f \in X^{\prime}$ the function $g \in G \mapsto f(\pi(g) x)$ is continuous on $G$;

2) for every measure $\alpha \in \mathcal{M}_{\sigma}(G)$ there exists an $X^{\prime}$-weakly continuous operator $\varphi(\alpha)$ : $X \rightarrow X$ such that

$$
f(\varphi(\alpha(x)))=\alpha(f(\pi(\cdot) x)), \quad x \in X, f \in X^{\prime}, \alpha \in \mathcal{M}_{\sigma}(G)
$$

It was shown in [14] that if $X$ is a quasicomplete barrelled space, then the second condition here can be omitted. This simplifies the construction, but it remains not clear in what sense $\mathcal{M}_{\sigma}(G)$ must be a Hopf algebra.

2. The situation becomes simpler if $G$ is supposed to be a real Lie group or a complex Stein group. In these cases the algebras $\mathcal{E}^{\prime}(G)$ of distributions with compact support and $\mathcal{O}^{\prime}(G)$ of analytical functionals respectively can be considered as group algebras, because

1) the smooth and the holomorphic actions of $G$ on $X$, defined by analogy, correspond to the continuous actions of $\mathcal{E}^{\prime}(G)$ and $\mathcal{O}^{\prime}(G)$, and

2 ) if $G$ is $\sigma$-compact, then $\mathcal{E}(G)$ and $\mathcal{O}(G)$ are Fréchet spaces and Hopf algebras with respect to the usual projective tensor product $\widehat{\otimes}$.

The last observation gives us hope that algebras $\mathcal{E}^{\prime}(G)$ and $\mathcal{O}^{\prime}(G)$ (i.e. dual spaces for $\mathcal{E}(G)$ and $\mathcal{O}(G))$ must be Hopf algebras with respect to some tensor product, but no accurate formulations of this were suggested in literature.

3. On the contrary, the situation becomes completely incomprehensible if $G$ is an affine algebraic group (over $\mathbb{C}$, for example). How can we define its group algebra $\mathbb{C} G$ in such a way that regular (or, in other words, polynomial) actions of $G$ on a vector space $X$ correspond to actions of $\mathbb{C} G$ on $X$ ? Strange thing, but this natural question also seems not to have been considered yet.

4. Let us note, finally, that the other classical algebras pretending to the role of group algebra for an arbitrary locally compact group:

- the algebra $L_{1}(G)$ of summable functions on $G$ [8],

- the $C^{*}$-algebra $C^{*}(G)$ of $G[8]$, and

- the von Neumann algebra $\operatorname{VN}(G)$ of $G[10]$ (or $\mathcal{L}(G)$ in the notations of [9]),

are not, formally speaking, Hopf algebras in the categorial sense.

The idea we have in mind is the following. There are, of course some generalizations of the notion of Hopf algebra, namely, Hopf $C^{*}$-algebras [16] and Hopf-von Neumann 
algebras [9] turning $C^{*}(G)$ and $\mathrm{VN}(G)$ respectively into "generalized Hopf algebras". But these approaches look not quite natural.

The simplest way to generalize Hopf algebras is, obviously, the one used in the theory of Quantum Groups, where topological Hopf algebras are defined as the ones that arise after replacing the algebraic tensor product $\otimes$ in the usual definition of Hopf algebra with another tensor product - let us denote it by $\otimes-$ which is defined, for example, as the completion of $\otimes$ under the $h$-adic topology (in the situation when the ring of formal power series $k[[h]]$ is used as the ground ring) [7]. So the next natural step in this direction should evidently be the following

Categorial definition of Hopf algebras. Let $(\mathcal{K}, \otimes)$ be a symmetric monoidal category. A Hopf algebra in $(\mathcal{K}, \bigotimes)$ is an object $H \in \mathcal{K}$ equipped with five morphisms $\mu: H \otimes H \rightarrow H$ (multiplication), $\varepsilon: \mathbb{C} \rightarrow H$ (unit), $\varkappa: H \rightarrow H \otimes H$ (comultiplication), $\iota: H \rightarrow \mathbb{C}$ (counit), $\sigma: H \rightarrow H$ (antipode) such that the usual claims figuring in the definition of Hopf algebra hold if we replace the algebraic tensor product $\otimes$ with the tensor product $\otimes$ of our category.

The problem with the algebras $L_{1}(G), C^{*}(G), \mathrm{VN}(G)$ is that in those cases either the unit (or the counit) is absent, or we are forced to use different tensor products for the multiplication and for the comultiplication.

As you guess, the reason why we began to speak about this is that stereotype theory gives common categorial constructions for some (we should say, main) cases mentioned above, namely for $\mathbf{1 , 2 , 3}$. An important side effect of this approach is that simultaneously it provides a natural solution of another standard problem in Quantum Group theory [6]: we obtain a convenient duality theory for infinite-dimensional Hopf algebras, which turns out here to be a special case of the classical Pontryagin-van Kampen duality.

4.1. Stereotype algebras, Hopf algebras and group algebras. As the category Ste has two natural tensor products- $\circledast$ and $\odot$ - there are two natural ways to define stereotype algebras:

- a projective stereotype algebra is a monoid $(A, \alpha, \varepsilon)$ in the category $(\mathfrak{S t e}, \circledast)$; a morphism of projective stereotype algebras is a morphism of such monoids;

- an injective stereotype algebra is a monoid $(A, \alpha, \varepsilon)$ in the category $(\mathfrak{S t e}, \odot)$; a morphism of injective stereotype algebras is a morphism of such monoids.

The first one of these two formal constructions has a convenient translation in topological terms:

Topological definition of stereotype algebra. A projective stereotype algebra (or, for short, stereotype algebra) can be defined equivalently as a stereotype space $A$ with the structure of unital associative algebra over $\mathbb{C}$, for which the multiplication operation is continuous in the following sense: for every compact set $K \subset A$ and for every net $x_{i}$ tending to zero in $A$ the nets $a \cdot x_{i}$ and $x_{i} \cdot a$ tend to zero in $A$ uniformly with respect to $a \in K$ :

$$
x_{i} \underset{i \rightarrow \infty}{\longrightarrow} 0 \quad \Longrightarrow \quad\left(a \cdot x_{i} \underset{i \rightarrow \infty}{\stackrel{a \in K}{\rightrightarrows}} 0, \quad \& \quad x_{i} \cdot a \underset{i \rightarrow \infty}{\stackrel{a \in K}{\rightrightarrows}} 0\right) .
$$


A morphism of stereotype algebras is an arbitrary continuous linear multiplicative map preserving the unit.

It is not difficult to see that every Fréchet algebra is stereotype. Besides this the following list gives an impression of the other

Examples of stereotype algebras:

- algebra of operators $\mathcal{L}(X):=\operatorname{Mor}(X, X)$ on a stereotype space $X$;

- function algebras:

$\mathcal{C}(M)$ algebra of (all) continuous functions on a paracompact locally compact topological space $M$ (with the topology of uniform convergence on compact sets);

$\mathcal{E}(M)$ algebra of smooth functions on a smooth manifold $M$ (with the usual topology);

$\mathcal{O}(M)$ algebra of holomorphic functions on a Stein maniofold $M$ (with the topology of uniform convergence on compact sets);

$\mathcal{R}(M)$ algebra of regular functions (=polynomials) on an affine algebraic manifold $M$ (with the strongest locally convex topology);

- group algebras (dual spaces for function algebras on groups):

$\mathcal{C}^{\star}(G)$ algebra of measures with compact support on a locally compact group $G$ (with the topology of uniform convergence on compact sets in $\mathcal{C}(G))$;

$\mathcal{E}^{\star}(G)$ algebra of distributions with compact support on a real Lie group $G$ (with the topology of uniform convergence on compact sets in $\mathcal{E}(G)$ );

$\mathcal{O}^{\star}(G)$ algebra of analytical functionals on a Stein group $G$ (with the topology of uniform convergence on compact sets in $\mathcal{O}(G))$;

$\mathcal{R}^{\star}(G)$ algebra of currents on an affine algebraic group $G$ (with the topology of uniform convergence on compact sets in $\mathcal{R}(G))$.

THEOREM 4. For every stereotype space $X$ the diagrams
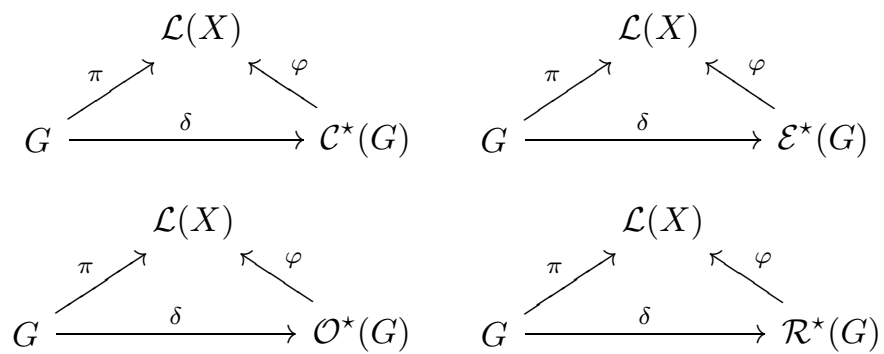

(where $\delta$ are natural embeddings as delta-functions) establish a one-to-one correspondence between

- continuous actions of an arbitrary locally compact group $G$ on $X$ and morphisms of stereotype algebras $\varphi: \mathcal{C}^{\star}(G) \rightarrow \mathcal{L}(X)$;

- smooth actions of an arbitrary real Lie group $G$ on $X$ and morphisms of stereotype algebras $\varphi: \mathcal{E}^{\star}(G) \rightarrow \mathcal{L}(X)$; 
- holomorphic actions of an arbitrary Stein group $G$ on $X$ and morphisms of stereotype algebras $\varphi: \mathcal{O}^{\star}(G) \rightarrow \mathcal{L}(X)$;

- regular actions of an arbitrary affine algebraic group $G$ on $X$ and morphisms of stereotype algebras $\varphi: \mathcal{R}^{\star}(G) \rightarrow \mathcal{L}(X)$.

\section{Theorem 5.}

- $\mathcal{C}^{\star}(G)$ is a Hopf algebra with respect to $\circledast($ and $\mathcal{C}(G)$ is a Hopf algebra with respect to $\odot)$.

- $\mathcal{E}^{\star}(G), \mathcal{O}^{\star}(G), \mathcal{R}^{\star}(G)$ (and $\mathcal{E}(G), \mathcal{O}(G), \mathcal{R}(G)$ ) are Hopf algebras with respect to $\circledast$ and $\odot$.

4.2. Algebras with reflection. Let $X$ be a stereotype space and let $\mathcal{L}^{\star}(X)$ denote the dual stereotype space for the stereotype algebra $\mathcal{L}(X)$ of operators on $X$. For every $x \in X$ and $f \in X^{\star}$ let us define a one-dimensional operator $f \odot x \in \mathcal{L}(X)$ and an elementary tensor $f \circledast x \in \mathcal{L}^{\star}(X)$ as follows:

$$
\begin{array}{r|lr}
f \odot x \in \mathcal{L}(X) & (f \odot x)(y)=f(y) \cdot x & (y \in X), \\
f \circledast x \in \mathcal{L}^{\star}(X) & (f \circledast x)(\varphi)=f(\varphi(x)) & (\varphi \in \mathcal{L}(X)) .
\end{array}
$$

THEOREM 6. There is a unique linear continuous map, turning $f \circledast x$ into $f \odot x$

$$
@: \mathcal{L}^{\star}(X) \rightarrow \mathcal{L}(X) \quad \mid \quad f \circledast x \stackrel{@}{\mapsto} f \odot x \quad\left(x \in X, f \in X^{\star}\right),
$$

and it satisfies the following two identities (where $A=\mathcal{L}(X)$ ):

$$
\begin{aligned}
& \text { (i) } @(a \cdot u \cdot b)=a \cdot @ u \cdot b, \quad\left(a, b \in A, u \in A^{\star}\right) \\
& \text { (ii) } \quad u(@ v)=v(@ u), \quad\left(u, v \in A^{\star}\right)
\end{aligned}
$$

Definition. If $A$ is a stereotype algebra, then a linear continuous map @ : $A^{\star} \rightarrow A$ satisfying (i) and (ii) is called a reflection on $A$.

EXAMPLES:

- $\mathcal{L}(X)$ for an arbitrary stereotype space $X$;

- $\mathcal{C}^{\star}(G), \mathcal{E}^{\star}(G)$, if $G$ is a compact group:

$$
@_{G} u(v)=\int_{G} u\left(t^{-1}\right) \cdot v(t) d t, \quad u, v \in \mathcal{C}(G), \mathcal{E}(G) .
$$

- $\mathcal{O}^{\star}(G), \mathcal{R}^{\star}(G)$, if $G$ is a reductive group (i.e. a complexification of a compact real Lie group $K)$ :

$$
@_{G} u(v)=\int_{K} u\left(t^{-1}\right) \cdot v(t) d t, \quad u, v \in \mathcal{O}(G), \mathcal{R}(G) .
$$

4.3. Reflections and representation theory. A morphism of algebras with reflections $\varphi$ : $\left(A, @_{A}\right) \rightarrow\left(B, @_{B}\right)$ is a morphism of stereotype algebras $\varphi: A \rightarrow B$ such that the following diagram is commutative:

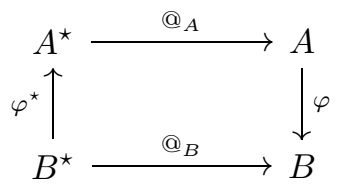


The following two results give an impression of what an unusual picture arises when we look at representation theory from the point of view of stereotype theory.

THEOREM 7. Suppose a compact group $G$ acts continuously on a finite-dimensional space $X$ :

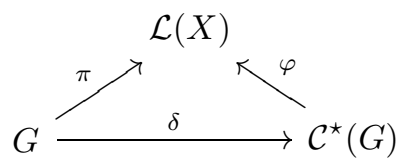

Then the following conditions are equivalent:

(i) the representation $\pi$ is irreducible;

(ii) $\varphi$ is a morphism of algebras with reflections $\left(\mathcal{C}^{\star}(G), @_{G}\right) \rightarrow\left(\mathcal{L}(X), \frac{1}{\operatorname{dim} X} @_{X}\right)$ :

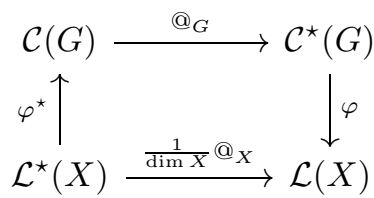

(iii) for every reflection $@_{1}$ on $\mathcal{L}(X)$ there is a reflection $@_{2}$ on $\mathcal{C}(G)$ such that $\varphi$ is a morphism of algebras with reflections $\left(\mathcal{C}^{\star}(G), @_{2}\right) \rightarrow\left(\mathcal{L}(X), @_{1}\right)$ :

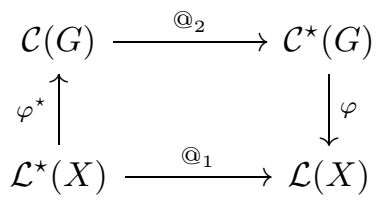

THEOREM 8. If $X$ is finite-dimensional, then $\left(\mathcal{L}(X), @_{X}\right)$ is a simple algebra with reflection in the following sense: every morphism $\varphi:\left(\mathcal{L}(X), @_{X}\right) \rightarrow\left(B, @_{B}\right)$ of algebras with reflection is an isomorphism automatically.

\section{Notion 2: approximation property}

5.1. Stereotype approximation property. We say that a stereotype space $X$ has the stereotype approximation property (S.A.P.) if the identity operator $1 \in \mathcal{L}(X)$ can be approximated in $\mathcal{L}(X)$ by operators of finite rank. This property lies between the classical approximation property and the existence of basis in $X$ :

\begin{tabular}{|c|c|c|c|c|}
\hline basis & $\Longrightarrow$ & $\begin{array}{c}\text { stereotype } \\
\text { approximation property }\end{array}$ & $\Longrightarrow$ & $\begin{array}{c}\text { classical } \\
\text { approximation property }\end{array}$ \\
\hline
\end{tabular}

The following theorem illustrates our statement that stereotype theory allows one to simplify Analysis by reducing the number of its counterexamples. It is well-known that if a Banach space $X$ has the classical approximation property, then the same is not necessarily true for its Banach space of operators $B(X)$ : A. Szankowski showed in [15] that the space $B(H)$ of bounded operators on a Hilbert space $H$ is a counterexample. In stereotype theory such counterexamples disappear:

THEOREM 9. If $X, Y$ have the stereotype approximation property, then $\operatorname{Mor}(X, Y), X \circledast Y$ and $X \odot Y$ have the stereotype approximation property as well. In particular, if $X$ has the 
stereotype approximation property, then $\mathcal{L}(X)$ has the stereotype approximation property as well.

5.2. "Absolute homology theory". In the usual approach to the homology theory of topological algebras - the relative homology theory [11] — we fix some class of epimorphisms (monomorphisms) called admissible, and then projective (injective) modules are defined as those for which the corresponding functors turn every admissible epimorphism (monomorphism) into an epimorphism in the Abelian category $\mathfrak{L} \mathfrak{i n}$ of linear spaces over $\mathbb{C}$ (not endowed with any topology). It turns out that for the stereotype algebras, one can apply another approach, called the absolute homology theory. Its idea is that, first, all epimorphisms (monomorphisms) in ${ }_{A} \mathfrak{S t e}$ are considered to be admissible, and, second, the projectivity and the injectivity are defined as those properties of modules for which the corresponding functors turn epimorphisms and monomorphisms in ${ }_{A} \mathfrak{S t e}$ into epimorphisms in the non-Abelian category $\mathfrak{S t e}$ of stereotype spaces. It turns out that in this "exotic" situation it is possible to compute homological dimensions and, what is strange, the arising results look very much like those from the relative homology theory.

Definition. Let $X$ be a stereotype module over $A$. We say that

- $X$ is approximatively projective if for every $A$-epimorphism $\varphi: E \rightarrow F$ and for each $A$-morphism $\chi: X \rightarrow F$ the following diagram "can be added approximatively":

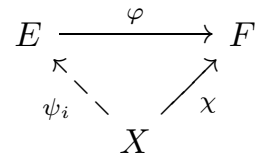

$$
\text { (i.e. } \left.\exists \psi_{i}: X \rightarrow E \quad \varphi \circ \psi_{i} \underset{i \rightarrow \infty}{\stackrel{\operatorname{Mor}(X, F)}{\longrightarrow}} \chi\right)
$$

- $X$ is approximatively injective if for every $A$-monomorphism $\varphi: E \rightarrow F$ and for each $A$-morphism $\chi: F \rightarrow X$ the following diagram "can be added approximatively":

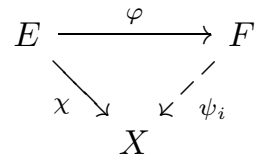

$$
\text { (i.e. } \left.\exists \psi_{i}: X \rightarrow E \quad \psi_{i} \circ \varphi \stackrel{\operatorname{Mor}(E, X)}{\underset{i \rightarrow \infty}{\longrightarrow}} \chi\right)
$$

THEOREM 10. If $A=\mathbb{C}$, then $X$ is approximatively projective $\Longleftrightarrow X$ is approximatively injective $\Longleftrightarrow X$ has the stereotype approximation property.

Definitions.

- A projective resolution of a stereotype $A$-module $X$ is defined as a complex

$$
0 \longleftarrow X \stackrel{\varphi_{0}}{\longleftarrow} P_{0} \stackrel{\varphi_{1}}{\longleftarrow} P_{1} \longleftarrow \ldots
$$

where $P_{i}$ are absolutely projective $A$-modules and $\operatorname{Ker} \varphi_{i}=\operatorname{Im} \varphi_{i+1}$.

- An injective resolution is defined as a complex

$$
0 \longrightarrow X \stackrel{\varphi_{0}}{\longrightarrow} Q_{0} \stackrel{\varphi_{1}}{\longrightarrow} Q_{1} \longrightarrow \ldots
$$

where $Q_{i}$ are absolutely injective $A$-modules and $\operatorname{Im} \varphi_{i}=\operatorname{Ker} \varphi_{i+1}$.

- A homological dimension $\operatorname{dh} A$ of $A$ is the minimal length of projective and injective resolutions for modules $X$ over $A$. 


\section{THEOREM 11.}

- $\operatorname{dh} \mathcal{L}(X)=0 \Longleftrightarrow X$ has the stereotype approximation property.

- $\operatorname{dh} \mathcal{C}(M)=0 \Longleftrightarrow M$ is discrete.

- $\operatorname{dh} \mathcal{C}^{\star}(G)=0 \Longleftrightarrow G$ is compact.

5.3. Modules with the stereotype approximation property. Let $X$ be a module over an algebra $A$. What are one-dimensional endomorphisms of $X$ over $A$ ?

$$
f \underset{A}{\odot} x: X \rightarrow X \quad\left(x \in X, f \in X^{\star}\right) .
$$

An interesting observation is that if $A$ has a reflection @, then these objects can be defined by the formula

$$
f \underset{A}{\odot_{A}} x(y)=@ f_{A}(y) \cdot x, \quad \text { where } \quad f_{A}: X \rightarrow A^{\star} \quad \mid \quad f_{A}(y)(a)=f(a \cdot y) .
$$

Definition. Let $X$ be a stereotype module over a stereotype algebra $A$. Its space of endomorphisms

$$
A^{!}(X)=\operatorname{End}_{A}(X)
$$

is called the commutant of the algebra $A$ in the module $X$. It turns out that the commutant $A^{!}(X)$ is always a stereotype algebra with respect to the composition $\varphi \circ \psi$.

THEOREM 12. If $(A, @)$ is an algebra with reflection, then for every A-module $X$ the commutant $A^{!}(X)=\operatorname{End}_{A}(X)$ has a reflection @! uniquely defined by the formula

$$
@^{!}: \operatorname{End}_{A}(X)^{\star} \rightarrow \operatorname{End}_{A}(X) \quad \mid \quad f \underset{A}{\circledast} x \stackrel{@ !}{\mapsto} f \underset{A}{\odot} x,
$$

where $f \underset{A}{\circledast} x$ is defined in the same way as $f \circledast x:(f \underset{A}{\circledast} x)(\varphi)=f(\varphi(x)), \varphi \in \operatorname{End}_{A}(X)$. DEFINITIONS.

- Morphisms of finite rank over ( $A$, @) are finite sums of one-dimensional morphisms: $\psi=\sum_{i=1}^{n} f_{i} \odot_{A} x_{i}$.

- $X$ has the stereotype approximation property (S.A.P.) over $(A, @) \Longleftrightarrow$ the identity morphism $1 \in \operatorname{End}_{A}(X)$ can be approximated in $\operatorname{End}_{A}(X)$ by morphisms of finite rank over $(A, @)$.

- $A$ acts effectively on $X \Longleftrightarrow\{(\forall x \in X \quad a \cdot x=0) \Rightarrow a=0\} \Longleftrightarrow \operatorname{Ker}\{A \rightarrow$ $\mathcal{L}(X)\}=0$

- A reflection @ : $A^{\star} \rightarrow A$ is exact $\Longleftrightarrow \operatorname{Ker} @=0 \Longleftrightarrow \operatorname{Im} @=A$.

- $A$ is a simple algebra $\Longleftrightarrow$ every non-zero two-sided ideal $I$ is dense in $A$.

EXAMPLE. The following conditions are equivalent:

(i) $\mathcal{L}(X)$ is a simple algebra;

(ii) its natural reflection $@: \mathcal{L}^{\star}(X) \rightarrow \mathcal{L}(X)$ is exact;

(iii) $X$ has the stereotype approximation property.

The following theorem shows that the operation $A \mapsto A^{!}(X)$ of taking commutant establishes a connection between the stereotype approximation property, effective actions and exact reflections: 


\section{THEOREM 13.}

- If $X$ has the stereotype approximation property over (A,@), then the commutant $\left(\operatorname{End}_{A}(X), @ !\right)$ is an algebra with an exact reflection, and acts effectively on $X$.

- If $(A, @)$ is an algebra with an exact reflection, and acts effectively on $X$, then $X$ has the stereotype approximation property over the commutant $\left(\operatorname{End}_{A}(X)\right.$, @!).

5.4. Bicommutant theorems. Let $X$ be a module over $A$. The commutant of the algebra $A^{!}(X)=\operatorname{End}_{A}(X)$ in the module $X$, is called the bicommutant of the algebra $A$ in the module $X$ :

$$
A^{! !}(X)=\left[A^{!}(X)\right]^{!}(X)=\operatorname{End}_{\operatorname{End}_{A}(X)}(X),
$$

Like in the Pure Algebra, there is a natural map of $A$ into its bicommutant:

$$
A \rightarrow A^{! !}(X)=\operatorname{End}_{\operatorname{End}_{A}(X)}(X)
$$

\section{THEOREM 14.}

- If $X$ is a left stereotype ideal in a simple algebra $A$, then $A$ is dense in its bicommutant $A^{! !}(X)$.

- If $(A, @)$ is an algebra with an exact reflection, and $A$ acts effectively on $X$, then $A$ is dense in its bicommutant $A^{! !}(X)$.

6. Open problems. In conclusion let us speak about some open problems in stereotype theory.

6.1. Stereotype Harmonic Analysis. A typical problem in Harmonic Analysis is the description of all $G$-modules for a given group $G$. Having in mind the correspondence between the actions of $G$ and of its group algebra $k G$, we can generalize this as follows.

Problem A. For a given algebra $A$ we wish to describe all A-modules.

Presumptuous as this declaration seems to be, we have some reasons to utter it. The fact is that problems of that kind, being transcendental for most situations in classical Analysis are not so hopeless in stereotype theory. For example, if $X$ is a Banach space, then it is apparently impossible to describe all (Banach) modules over the (Banach) algebra $B(X)$ of operators on $X$. The only exception is the special case, when $X$ is finite-dimensional - then we have the purely algebraic fact that all the modules $M$ over $B(X)=\mathcal{L}(X)$ have the form

$$
M=E \otimes X \quad \text { (where } E \text { are suitable vector spaces). }
$$

It is amazing, but this proposition has a natural generalization in stereotype theory. To formulate it we should make some preparations. Suppose $E$ and $X$ are stereotype spaces. It is known that their stereotype tensor products $E \circledast X$ and $E \odot X$ are connected with each other through a natural linear continuous map named Grothendieck transformation $($ see $[2])$ :

$$
\text { @ : } E \circledast X \rightarrow E \odot X .
$$

It can be defined as a unique linear continuous map turning the elementary tensor $e \circledast x$ into the elementary tensor $e \odot x$ (the definitions of $e \circledast x$ and $e \odot x$ are the same as those 
in Subsection 5.3):

$$
e \circledast x \stackrel{@}{\mapsto} e \odot x \quad(e \in E, x \in X) .
$$

In the special case when $E=X^{\star}$ this map coincides with the natural reflection on $\mathcal{L}(X)$ defined in Subsection 5.3 (and this explains why we use the same symbol @ for reflections and for Grothendieck transformations):

$$
\text { @ : } X^{\star} \circledast X=\mathcal{L}^{\star}(X) \rightarrow \mathcal{L}(X)=X^{\star} \odot X .
$$

If $X$ has the stereotype approximation property, then it turns out that @ : $E \circledast X \rightarrow E \odot X$ is injective and has dense range in $E \odot X$. The following theorem asserts that every $\mathcal{L}(X)$-module $M$ in this case lies between $E \circledast X$ and $E \odot X$ for some appropriate $E$ :

$$
E \circledast X \subseteq M \subseteq E \odot X .
$$

THEOREM 15. Let $X$ be a stereotype space with the stereotype approximation property. Then for every left stereotype module $M$ over the stereotype algebra $\mathcal{L}(X)$ there exists a unique stereotype space $E$ and two natural bimorphisms $\rho, \sigma$ of stereotype $\mathcal{L}(X)$-modules (i.e., $\mathcal{L}(X)$-linear continuous injective maps with dense range) such that the following diagram is commutative:

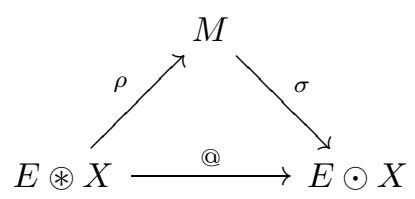

Corollary. Let $X$ be a nuclear Fréchet space with a basis. Then for every Fréchet module $M$ over the stereotype algebra $\mathcal{L}(X)$ there is a unique Fréchet space $E$ such that

$$
M \cong E \widehat{\otimes} X .
$$

From the philosophical point of view, these results can be considered as supplementary evidence of our common declaration that stereotype theory simplifies Functional Analysis like nonconstructive proofs simplify Mathematical Analysis. And this gives us hope that Problem A can have natural solution for many stereotype algebras, in particular, for those listed in Subsection 4.1. One special question here is: what is the structure of $\mathcal{L}(X)$-modules $M$, when $X$ fails the stereotype approximation property?

6.2. Role of reflections in Harmonic Analysis. The results of Subsection 4.3 show that reflections play an intriguing (and absolutely uninvestigated) role in Harmonic Analysis. Theorems 7 and 8 mean, in particular, that we can try to reconstruct Harmonic Analysis in such a way that it is turned into an investigation of a category of stereotype algebras endowed with the supplementary structure - reflection: Theorem 8 explains how we should define simple objects in this category, and Theorem 7 shows that every object can be decomposed in some reasonable sense into simple ones.

There are, nevertheless, some unclear places in this program. One of them is that we do not know what we should do with the group algebras of non-compact (and non-reductive) groups. The fact is that they apparently have no non-zero reflections, and because of that the theory becomes trivial in this situation. So we shall formulate the second important problem of stereotype theory as follows. 
PROBlem B. Is it possible to describe irreducible representations of non-compact groups in terms of reflections?

The difference between compact and non-compact situations in group algebras suggests, in particular, the idea that amenability must also have a natural description in stereotype langauge:

Problem C. Is it possible to describe amenability by means of reflections (or by other means of stereotype theory)?

6.3. Intrinsic problems of stereotype theory. Apart from those problems concerning applications of the stereotype theory, there are, of course, many blank spots inside the theory. One of them is that we know almost nothing about the topology of $\mathcal{L}(X)$ (not counting the fact that this space is stereotype $\left.{ }^{1}\right)$. What kind of topological vector spaces are $\mathcal{L}(X)$ ? It is not difficult to see, for example, that $\mathcal{L}(X)$ cannot be a Banach space if $X$ is Banach (infinite-dimensional, of course). This becomes clear from the following

Lemma. If $\mathcal{L}(X)$ is barrelled, then $X$ and $\mathcal{L}(X)$ are Montel spaces.

Proof. Take $x \in X$ and $f \in X^{\star}$ such that $f(x)=1$. Then the maps

$$
\pi, \sigma: \mathcal{L}(X) \rightarrow \mathcal{L}(X) \quad \mid \quad \pi(\varphi)=\varphi \circ f \odot x, \quad \sigma(\varphi)=f \odot x \circ \varphi
$$

are projections in $\mathcal{L}(X)$ (i.e. $\pi^{2}=\pi$ and $\sigma^{2}=\sigma$ ) and $\operatorname{Im} \pi \cong X, \operatorname{Im} \sigma \cong X^{\star}$. Hence, if $\mathcal{L}(X)$ is barrelled, then $X$ and $X^{\star}$ are barrelled as well. The second condition mean that $X$ has Heine-Borel property, so $X$ must be a Montel space. This in its turn implies that $\mathcal{L}(X)$ must have Heine-Borel property, and thus, $\mathcal{L}(X)$ is a Montel space as well.

Strange thing, but the inverse implication does not look trivial:

Problem D. Suppose $X$ is a Montel space. Is its stereotype space of operators $\mathcal{L}(X)$ a Montel space as well?

Another blank spot looks unbecomingly challenging:

PROBLEM E. Is the stereotype approximation property equivalent to the classical approximation property?

\section{References}

[1] S. S. Akbarov, Stereotype spaces, algebras, homologies: An outline, in: Topological Homology, A. Ya. Helemskii (ed.), Nova Science Publishers, 2000, 1-29.

[2] S. S. Akbarov, Pontryagin duality in the theory of topological vector spaces and in topological algebra, Journal of Mathematical Sciences 113 (2003), 179-349.

\footnotetext{
${ }^{1}$ We should specify here (again, see details in [2]) that by definition $\mathcal{L}(X)$ is the pseudosaturation $(X: X)^{\Delta}$ of the space $X: X$ of linear continuous maps $\varphi: X \rightarrow X$ endowed with the compact-open topology. Our declaration that $\mathcal{L}(X)=(X: X)^{\Delta}$ is stereotype means only that $X: X$ becomes stereotype after applying the operation $\Delta$. But actually we do not even know whether this operation has to be applied-perhaps $X: X$ is already pseudosaturated (and, as a corollary, stereotype)?
} 
[3] S. S. Akbarov and E. T. Shavgulidze, On two classes of spaces reflexive in the sense of Pontryagin, Matematicheskij Sbornik 194 (2003), 3-26.

[4] P. S. Aleksandrov, An Introduction to Set Theory and to General Topology, Nauka, Moscow, 1977 (in Russian).

[5] V. A. Artamonov, V. N. Saliy, L. A. Skornjakov, L. N. Shevrin, and E. G. Shulgeifer, General Algebra, Nauka, Moscow, 1991 (in Russian).

[6] P. Bonneau, M. Flato, M. Gerstenhaber, and G. Pinczon, The hidden group structure of quantum groups: strong duality, rigidity and preferred deformations, Commun. Math. Phys. 161 (1994), 125-156.

[7] V. Chari and A. Pressley, A Guide to Quantum Groups, Cambridge University Press, 1995.

[8] J. Dixmier, Les $C^{*}$-algèbres et leurs représentations, Gauthier-Villars, 1969.

[9] M. Enock and J.-M. Schwartz, Kac Algebras and Duality of Locally Compact Groups, Springer Verlag, 1992.

[10] P. Eymard, L'algèbre de Fourier d'un groupe localement compact, Bull. Soc. Math. France 92 (1964), 181-236.

[11] A. Ya. Helemskii, Banach and Polynormed Algebras: General Theory, Representations, and Homologies, Nauka, Moscow, 1989 (in Russian).

[12] G. Kelly, Basic Concepts of Enriched Category Theory, London Math. Soc. Note Ser. 64, 1982.

[13] B. A. Kushner, Lectures on Constructive Mathematical Analysis, Translations of Mathematical Monographys 60, American Mathematical Society, 1985.

[14] G. L. Litvinov, Representations of groups in locally convex spaces and topological group algebras, Selecta Mathematica Sovietica 7 (1988), 101-182.

[15] A. Szankowski, B(H) does not have the approximation property, Act. Math. 147 (1981), 89-108.

[16] S. Vaes and A. Van Daele, Hopf $C^{*}$-algebras, Proc. London Math. Soc. 82 (2001), 337-384. 